\title{
Using the singular value decomposition to extract 2D correlation functions from scattering patterns
}

\section{Philipp Bender, Dominika Zákutná, Sabrina Disch, Lourdes Marcano, Diego Alba Venero and Dirk Honecker}

Acta Cryst. (2019). A75, 766-771

\section{IUCr Journals CRYSTALLOGRAPHY JOURNALS ONLINE}

Copyright (C) International Union of Crystallography

Author(s) of this article may load this reprint on their own web site or institutional repository provided that this cover page is retained. Republication of this article or its storage in electronic databases other than as specified above is not permitted without prior permission in writing from the IUCr.

For further information see http://journals.iucr.org/services/authorrights.html 


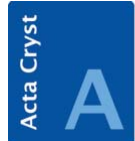

FOUNDATIONS

ADVANCES

ISSN 2053-2733

Received 27 March 2019

Accepted 21 June 2019

Edited by S. J. L. Billinge, Columbia University, USA

₹ Current address: Physics and Materials Science Research Unit, University of Luxembourg, 162A Avenue de la Faïencerie, L-1511 Luxembourg, Grand Duchy of Luxembourg.

Keywords: small-angle scattering; correlation function; 2D Fourier transform; anisotropic nanoparticles; singular value decomposition; anisotropic structures; nanoparticles; noise filtering.

Supporting information: this article has supporting information at journals.iucr.org/a

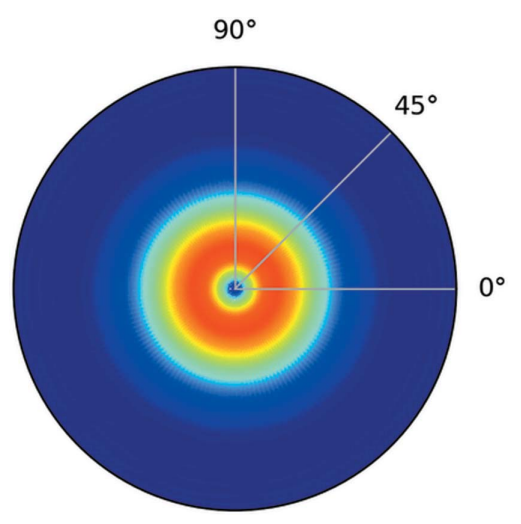

(C) 2019 International Union of Crystallography

\section{Using the singular value decomposition to extract 2D correlation functions from scattering patterns}

\author{
Philipp Bender, ${ }^{\text {a* }}$ Dominika Zákutná, ${ }^{\mathrm{b}, \mathrm{c}}$ Sabrina Disch, ${ }^{\mathrm{c}}$ Lourdes Marcano, ${ }^{\mathrm{d}, \mathrm{e}}$ Diego \\ Alba Venero ${ }^{f}$ and Dirk Honecker ${ }^{b} \neq$
}

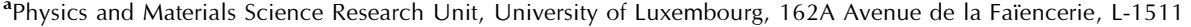
Luxembourg, Grand Duchy of Luxembourg, 'barge Scale Structures Group, Institut Laue-Langevin, 71 Avenue des Martyrs, F-38042 Grenoble, France, ' Department für Chemie, Universität zu Köln, Luxemburger Strasse 116, D-50939 Köln, Germany, ${ }^{\mathbf{d}}$ Helmholtz-Zentrum Berlin für Materialien und Energie, Albert-Einstein-Strasse 15, D-12489 Berlin, Germany, ${ }^{\mathbf{E}}$ Departamento Electricidad y Electrónica, Universidad del País Vasco - UPV/EHU, E-48940 Leioa, Spain, and IISIS Neutron and Muon Facility, Rutherford Appleton Laboratory, Chilton, OX11 0QX, UK. *Correspondence e-mail: philipp.bender@uni.lu
\end{abstract}

The truncated singular value decomposition (TSVD) is applied to extract the underlying 2D correlation functions from small-angle scattering patterns. The approach is tested by transforming the simulated data of ellipsoidal particles and it is shown that also in the case of anisotropic patterns (i.e. aligned ellipsoids) the derived correlation functions correspond to the theoretically predicted profiles. Furthermore, the TSVD is used to analyze the small-angle X-ray scattering patterns of colloidal dispersions of hematite spindles and magnetotactic bacteria in the presence of magnetic fields, to verify that this approach can be applied to extract model-free the scattering profiles of anisotropic scatterers from noisy data.

\section{Introduction}

Small-angle scattering of X-rays (SAXS) or neutrons (SANS) is routinely used to investigate the structural properties of various materials, such as nanostructured bulk samples (FritzPopovski, 2013, 2015; Michels, 2014) or nanoparticle ensembles (Reufer et al., 2010; Märkert et al., 2011; Hoffelner et al., 2015; Nack et al., 2018). In many cases, the detected 2D scattering patterns $I(\mathbf{q})$ (here $I$ is the scattering intensity and $\mathbf{q}$ the scattering vector on the $2 \mathrm{D}$ detector plane) are isotropic and data analysis is performed on the $1 \mathrm{D}$ azimuthal average $I(q)$, by either fitting it in reciprocal space with model functions or by transforming $I(q)$ to real-space correlation functions $C(r)$ via Fourier transforms (FT) (Pedersen, 1997). For the latter, a direct FT can be used (Michels et al., 2003) or, alternatively, an indirect FT (Glatter, 1977; Hansen, 2000; Bender et al., 2017). Often the pair distance distribution function $P(r)=C(r) r^{2}$ is determined, which gives direct information about the average shape, size and structure of the scattering units (Glatter, 1979; Svergun \& Koch, 2003). In the case of anisotropic nanostructures, the pattern $I(\mathbf{q})$ can be anisotropic, provided the nanostructures are preferentially aligned along a certain direction or texture axis (Heijden et al., 2004; Reufer et al., 2010; Märkert et al., 2011; Fritz-Popovski, 2013; Hoffelner et al., 2015; Nack et al., 2018). Also, in this case, data analysis can be performed either by adjusting the whole scattering pattern in reciprocal space (Alves et al., 2017) or by transforming the pattern via 2D FTs to the real-space 2D correlation functions (Fritz-Popovski, 2013, 2015; Mettus \& Michels, 2015). For the indirect 2D FT proposed by Fritz-Popovski (2013), regularization functionals are appended to the system matrix, which is 
necessary to obtain smooth profiles, but which can increase the computation times significantly. Here, we propose a similar but faster method, namely the truncated singular value decomposition (TSVD), to extract the underlying 2D correlation functions. We propose that this method could be used for live, model-free data analysis, e.g. in capillary flow devices (Nielsen et al., 2012), time-resolved nanoparticle oscillation (Bender et al., 2015) or rheo-SAXS/SANS experiments (Panine et al., 2003; Calabrese et al., 2016), and microfluidics (Stehle et al., 2013; Poulos et al., 2016; Adamo et al., 2018), where the noise reduction of the singular value decomposition (SVD) helps to enhance the signal.

\section{Methods}

\subsection{Singular value decomposition}

In polar coordinates the 2D scattering intensity $I\left(q_{y}, q_{z}\right)=I(q, \Theta)$ (the beam is along the $x$ direction, $\left.\mathbf{k} \| \mathbf{e}_{x}\right)$ is a function of the magnitude of the scattering vector $|\mathbf{q}|$ and the angle $\Theta$. With $i=N$ unique data points (i.e. pixels of the detector for a monochromatic source) it can be written

$$
I\left(q_{i}, \Theta_{i}\right)=\sum_{k=1}^{K} A_{i k} P\left(r_{k}, \varphi_{k}\right)
$$

where the angle $\varphi$ specifies the orientation of $\mathbf{r}$ in real space in the $y z$ plane. Here, the extracted 2D distribution function is $P(r, \varphi)=C(r, \varphi) r$ (Fritz-Popovski, 2013) and the matrix A has the elements (Mettus \& Michels, 2015)

$$
A_{i k}=\cos \left[q_{i} r_{k} \cos \left(\Theta_{i}-\varphi_{k}\right)\right] \Delta r_{k} \Delta \varphi_{k}
$$

Here, we usually assume a linear spacing for the predetermined $r$ and $\varphi$ vectors (i.e. $\Delta r$ and $\Delta \varphi=$ const.). By minimizing the deviation (i.e. performing a least-square fit)

$$
\chi^{2}=\sum_{i=1}^{N} \frac{\left[I\left(q_{i}, \Theta_{i}\right)-\sum_{k=1}^{K} A_{i k} P\left(r_{k}, \varphi_{k}\right)\right]^{2}}{\sigma_{i}^{2}}
$$

the 2D correlation function $P(r, \varphi)=C(r, \varphi) r$ can be determined. In the case of significant measurement uncertainties $\sigma_{i}$, however, this distribution will exhibit strong, unphysical oscillations. While performing the indirect FT, regularization matrices are appended (e.g. a Tikhonov regularization can be applied) to force smooth distributions (Fritz-Popovski, 2013), and various approaches exist to estimate the optimal value for the regularization parameter (Glatter, 1977; Hansen, 2000).

With the SVD the decomposition of the system matrix $\mathbf{A}=\mathbf{U S V}^{\mathrm{T}}$ is performed, where $\mathbf{U}$ and $\mathbf{V}$ are orthogonal $N \times N$ and $K \times K$ matrices, respectively, and $\mathbf{S}$ is an $N \times K$

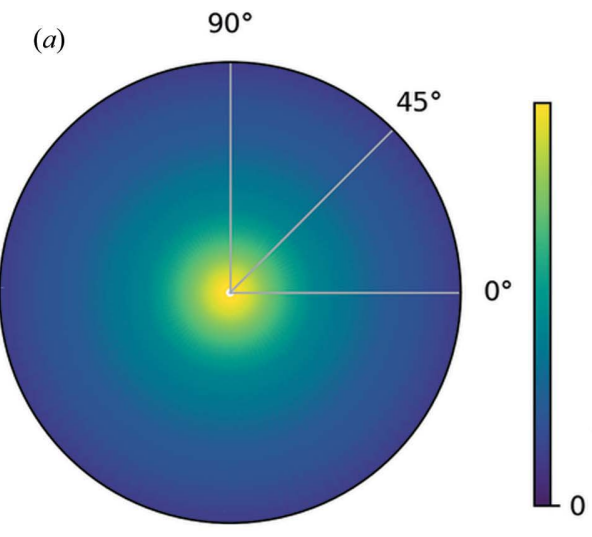

(b)

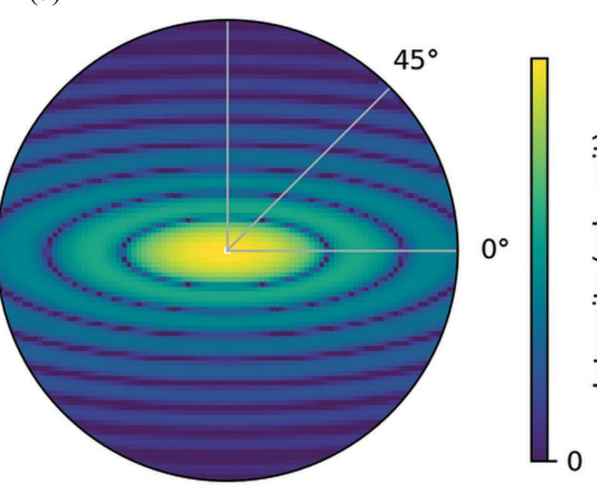

Figure 1

Determination of the 2D correlation functions by an SVD for a monodisperse ensemble of prolate rotational ellipsoids with an equatorial radius of $50 \mathrm{~nm}$ and a polar radius of $150 \mathrm{~nm}$. (a) Ellipsoids are randomly oriented (isotropic ensemble). $(b)$ Ellipsoids are aligned with their polar axis along $\Theta=$ $90^{\circ}$ (anisotropic ensemble). Left column: polar plot of the scattering pattern in logarithmic scale $\left(q_{\max }=0.2 \mathrm{~nm}^{-1}\right)$. Middle column: extracted $2 \mathrm{D}$ correlation function $P(r, \varphi)\left(r_{\max }=400 \mathrm{~nm}\right)$. Right column: correlation function along $\varphi=0^{\circ}$ and $90^{\circ}$, and in $(b)$ additionally the calculated cross sections for spheres with diameters of $100 \mathrm{~nm}$ and $300 \mathrm{~nm}$, respectively. 
matrix whose main diagonal elements $s_{i} \equiv s_{i i}$ are the singular values (all other values are zeros). The solution $P(r, \varphi)$ of the functional $\mathbf{A} P(r, \varphi)=I(q, \Theta)$ is

$$
P(r, \varphi)=\mathbf{V S}^{+} \mathbf{U}^{\mathrm{T}} I(q, \Theta)
$$

where $\mathbf{S}^{+}$has the reciprocal values $1 / s_{i}$ in its diagonal, and otherwise zeros. Using the full-rank matrices for the reconstruction of $P(r, \varphi)$ according to equation (4) should result in the same distribution as minimizing equation (3). In particular, very small $s_{i}$ values associated with noise result in strong oscillations of the reconstructed distribution. Thus, a smoothing of $P(r, \varphi)$ can be achieved by reducing the number of singular values $s_{i}$ that are considered for $\mathbf{S}^{+}$(Berkov et al., 2000) (i.e. singular values which are smaller than a certain threshold are set to zero). Alternatively, a TSVD can be performed (Hansen, 1987; Viereck et al., 2019), which further reduces the computation time compared with an SVD of the full-ranked matrices.

We will use the TSVD to analyze first the simulated scattering patterns of an ensemble of prolate ellipsoids and later the measured SAXS patterns of a colloidal dispersion of hematite spindles and of magnetotactic bacteria.

\subsection{Samples}

Details regarding the synthesis and characterization of the hematite spindles can be found in the work of Zákutná et al. (2019). Hematite nanospindles are weakly ferromagnetic and known to orient with their main axis perpendicular to an applied magnetic field (Reufer et al., 2010; Märkert et al., 2011; Roeder et al., 2015; Hoffelner et al., 2015; Nack et al., 2018). According to electron microscopy, the sample studied here has a length of the short spindle axes of around $56 \mathrm{~nm}$ and of the long axis of around $375 \mathrm{~nm}$. The SAXS measurements from the work of Zákutná et al. (2019), which we also analyze here, were performed at the ID02 instrument at the ESRF (Narayanan et al., 2018) on a dilute, i.e. non-interacting, dispersion of the nanospindles. A static homogeneous magnetic field up to $520 \mathrm{mT}$ was applied with an electromagnet in the horizontal direction perpendicular to the incoming beam.

A detailed study of the magnetotactic bacteria is presented by Orue et al. (2018). These bacteria contain on average about 15-20 iron oxide nanoparticles with diameters of around 40$50 \mathrm{~nm}$, which are typically arranged in nearly linear chains. Here we analyze a SAXS measurement of the same colloidal dipsersion of bacteria as presented in the work of Orue et al. (2018). This measurement was performed with a Xenocs Nano-InXider. The colloidal dispersion was filled into a quartz glass capillary and the bacteria aligned perpendicular to the incoming beam by positioning a small permanent magnet next to the capillary. With this setup only one full quadrant of the pattern is detected, but assuming symmetry the total $2 \pi$ pattern can be easily reconstructed.
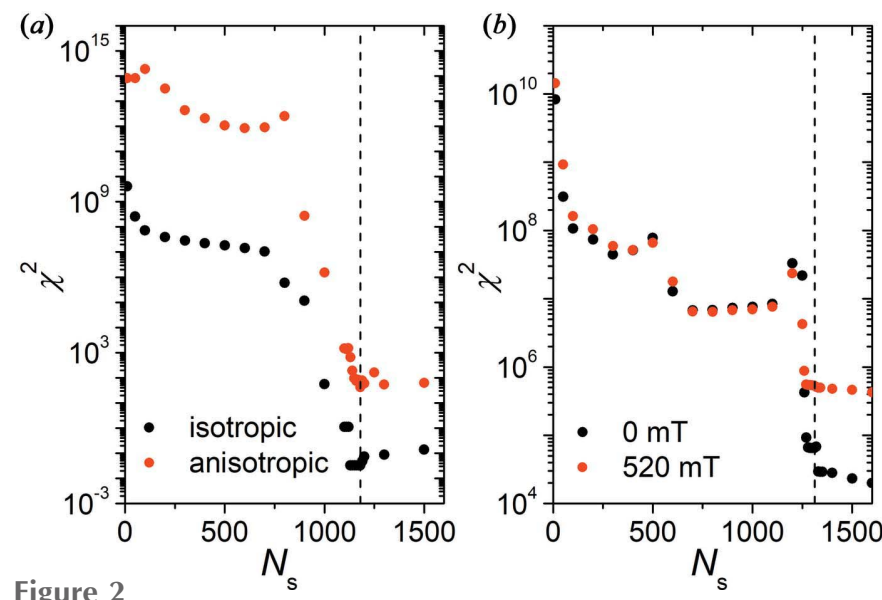

Error $\chi^{2}$ [equation (3)] for the fit of (a) the simulated data (Fig. 1) and $(b)$ the experimental data of the spindles (Fig. 3) at two different fields, with $N_{\mathrm{s}}$ being the number of singular values used for the reconstruction.

\section{Results}

\subsection{Simulated data}

To test the TSVD, we first analyze the calculated scattering patterns of rotational ellipsoids with an equatorial radius of $50 \mathrm{~nm}$ and a polar radius of $150 \mathrm{~nm}$ [left column in Figs. 1(a) and 1(b)]. In Fig. 1(a) (upper row) we analyze the isotropic ensemble and in Fig. 1(b) (lower row) the anisotropic ensemble. The simulated scattering patterns had $N=30480$ data points, and we calculated the system matrix A $(30480 \times$ 10224 matrix) for $101 r$ values (0-400 nm, $4 \mathrm{~nm}$ steps) and 144 $\varphi$ values $\left(2.5-360^{\circ}, 2.5^{\circ}\right.$ steps $)$. We then varied the number of singular values $N_{\mathrm{s}}$ considered for the reconstruction. Usually, $N_{\mathrm{s}}$ is much smaller than the dimensions of the system matrix, and thus the TSVD significantly reduces the computational complexity compared for example with the classical indirect FT (IFT) approaches. As shown in Fig. 2(a), the total error $\chi^{2}$ is significantly reduced by increasing $N_{\mathrm{s}}$ and exhibits a minimum at around $N_{\mathrm{s}}=1180$, which is marked by the dashed line [here we used $\sigma_{i}^{2}=I\left(q_{i}, \Theta_{i}\right)$ for the calculation of $\chi^{2}$, equation (3)]. Further increasing $N_{\mathrm{s}}$ does not further minimize the deviations, but will generate strong oscillations due to the restricted $q$ range (Qiu et al., 2004). In Fig. 1 we plot the reconstructed distributions for $N_{\mathrm{s}}=1180$, and in the case of the anisotropic ensemble compare them with the theoretically expected profiles $C(r) r$ (Fritz-Popovski, 2015), which we calculated for spheres with diameters of $100 \mathrm{~nm}$ and $300 \mathrm{~nm}$, respectively. The good agreement shows that the TSVD can be readily used for a fast determination of the underlying 2D correlation functions from 2D scattering patterns, at least in the case of smooth data.

\subsection{Experimental data}

3.2.1. Hematite spindles. In Fig. 3 we show the analysis results of the field-dependent scattering patterns of the colloidal dispersion of hematite spindles. In the upper row [Fig. 3(a)] we display the results for $0 \mathrm{mT}$ and in the lower row [Fig. 3(b)] the results for $\mu_{0} H=520 \mathrm{mT}$. For both, the isotropic and the anisotropic pattern, the SVD was performed 


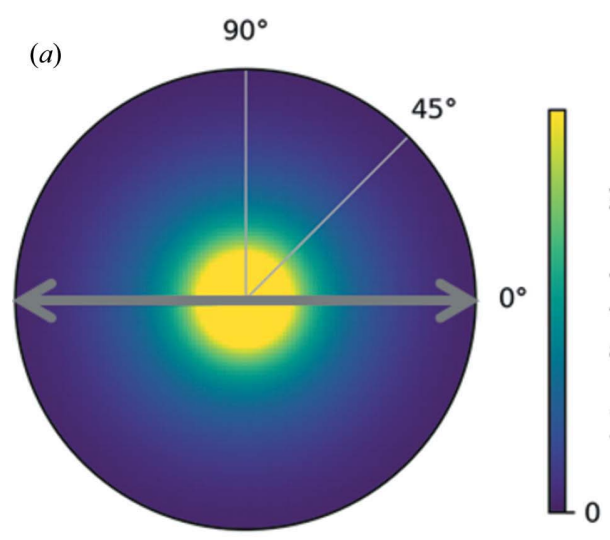

(b)

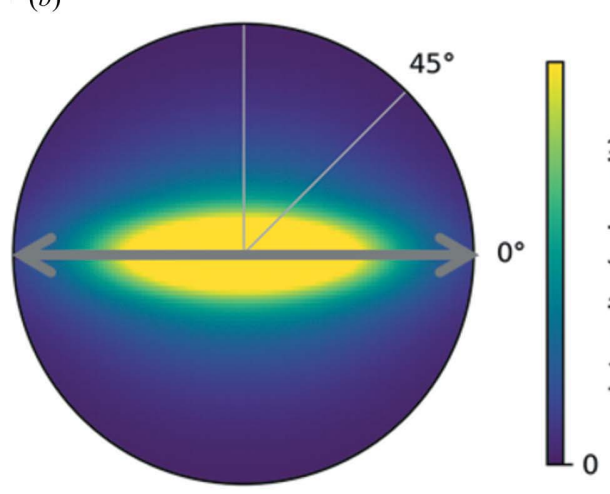

Figure 3
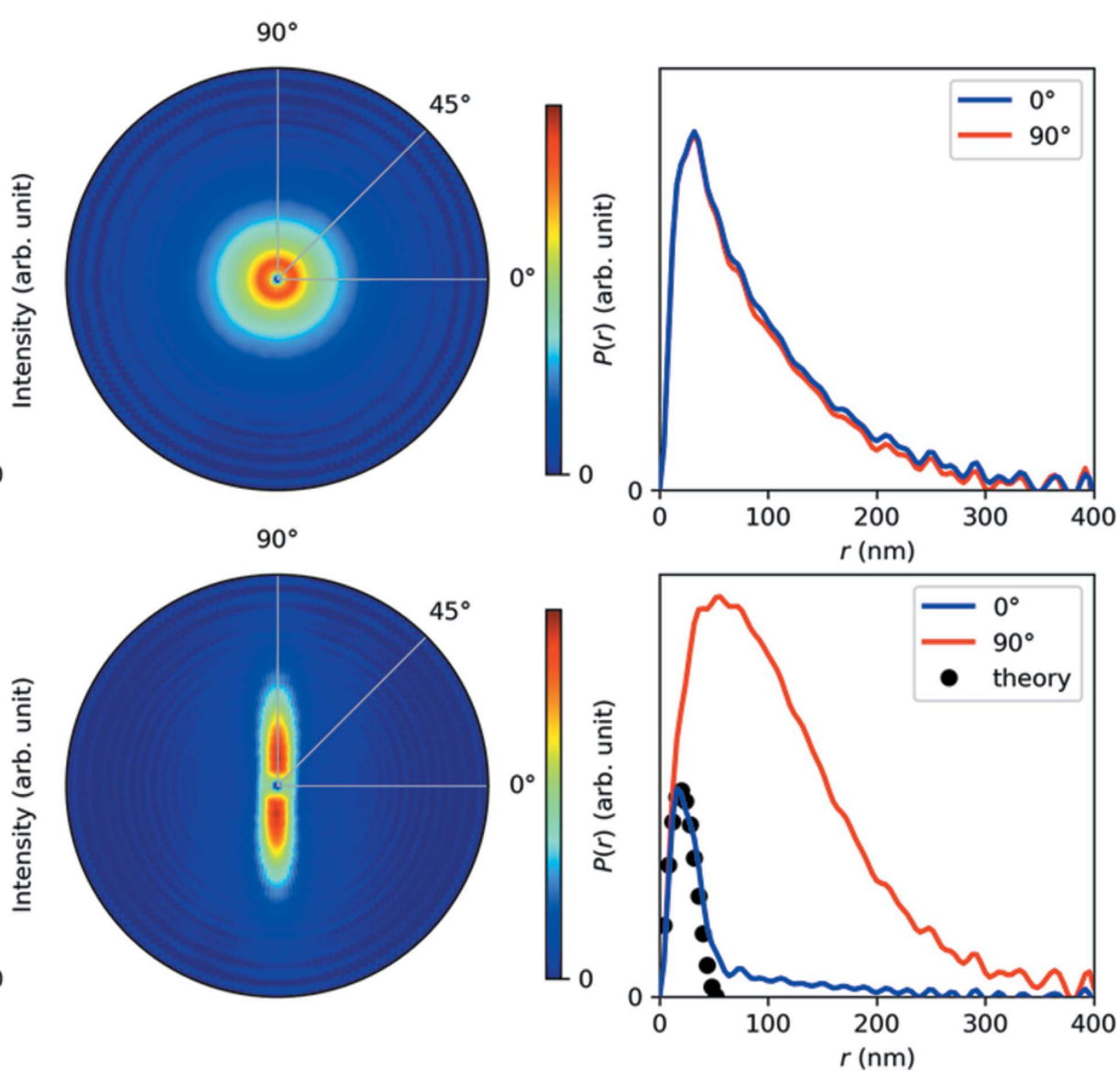

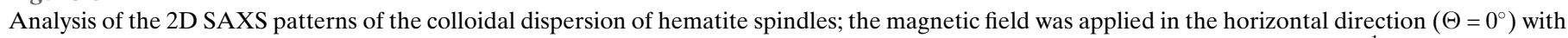

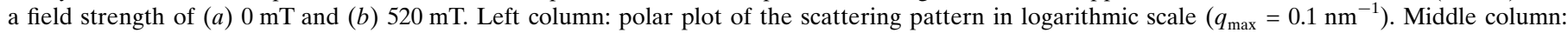

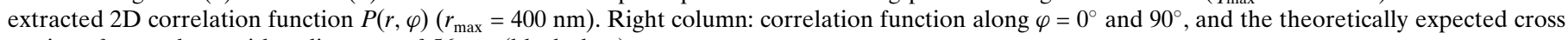
sections for a sphere with a diameter of $56 \mathrm{~nm}$ (black dots).

for $101 r$ values $(0-400 \mathrm{~nm})$ and $144 \varphi$ values $\left(2.5-360^{\circ}, 2.5^{\circ}\right.$ steps). The correlation functions plotted in Fig. 3 were determined for $N_{\mathrm{s}}=1270$, for which the total error was already close to the minimum [Fig. 2(b)]. For both cases the extracted correlation functions exhibit some oscillations, which increases significantly with increasing $N_{\mathrm{s}}$ (i.e. smaller singular values are associated with signal noise).

At $0 \mathrm{mT}$, the anisotropic particles are randomly oriented within the viscous matrix (water), and thus the scattering pattern and the derived correlation function are both isotropic. The extracted profile has a pronounced peak at around $50 \mathrm{~nm}$ and a long tail, and hence corresponds well to the expected profile of randomly oriented, shape-anisotropic nanoparticles (Svergun \& Koch, 2003).

At $520 \mathrm{mT}$, on the other hand, the particle moments are mostly oriented in the field direction. The dominance of the magnetocrystalline anisotropy leads to a net magnetic moment in the basal plane of the hematite spindles, i.e. perpendicular to the long particle axis (Reufer et al., 2010; Hoffelner et al., 2015; Nack et al., 2018). Therefore, the long spindle axes are oriented within the plane perpendicular to the field direction, which explains the anisotropy of both the scattering pattern and the extracted correlation function. Along $\varphi=90^{\circ}$ [right column of Fig. 3(b)] the profile indicates a maximum length of the spindles of around $400 \mathrm{~nm}$, whereas the profile along $\varphi=0^{\circ}$ agrees well with the expected correlation function for spindles with an equatorial diameter of $56 \mathrm{~nm}$. Thus, the extracted 2D correlation function is in good agreement with our previous results (Zákutná et al., 2019).

3.2.2. Magnetotactic bacteria. In Fig. 4 we show the highly anisotropic SAXS pattern of the aligned magnetotactic bacteria along the horizontal field direction. The extracted correlation function was determined, as before, for $101 r$ values $(0-200 \mathrm{~nm})$ and $144 \varphi$ values $\left(2.5-360^{\circ}, 2.5^{\circ}\right.$ steps $)$. The function plotted in Fig. 4 was determined for $N_{\mathrm{s}}=1000$, which shows that the particles are preferentially spheres (radially symmetric spots), and that the bacteria and thus the magnetosome chains are well aligned along the applied field showing no spreading of the spots in the azimuthal direction. From the profile along $\varphi=0^{\circ}$ (right column of Fig. 4) we can estimate the average center-to-center distance between the particles in the chain from the peak-to-peak distance to be around $50 \mathrm{~nm}$ and the particle size from the peak width to be around $40 \mathrm{~nm}$, which agrees well with previous results (Orue et al., 2018). Along $\varphi=90^{\circ}$ we only see the cross section of a single particle. However, for $r>40 \mathrm{~nm}$ the profile oscillates around zero. This indicates a variation of the scattering length density profile, which we attribute to a lower scattering length 


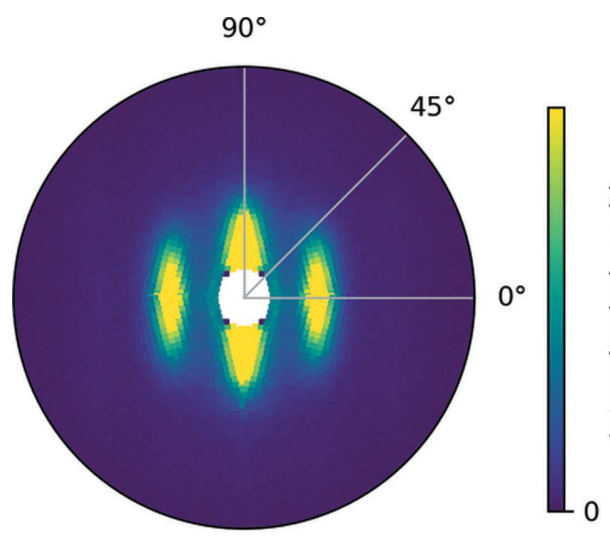

Figure 4

Analysis of the 2D SAXS pattern of the colloidal dispersion of magnetotactic bacteria; the magnetic field was applied in the horizontal direction $\left(\Theta=0^{\circ}\right)$. Left column: polar plot of the scattering pattern in logarithmic scale $\left(q_{\max }=0.5 \mathrm{~nm}^{-1}\right)$. Middle column: extracted 2D correlation function $P(r, \varphi)\left(r_{\max }=\right.$ $150 \mathrm{~nm})$. Right column: correlation function along $\varphi=0^{\circ}$ and $90^{\circ}$.

density in the vicinity of the particle (i.e. the bacteria) compared with the surrounding matrix (i.e. the water).

\section{Conclusions}

Here, we introduce the truncated singular value decomposition (TSVD) to extract the denoised 2D correlation functions from small-angle scattering patterns. First, we analyze simulated data of ellipsoidal particles and show that the derived correlation functions correspond well to the theoretically predicted profiles. Furthermore, we use this method to successfully extract the underlying 2D correlation functions from experimental SAXS patterns of colloidal dispersions of hematite spindles and magnetotactic bacteria. We emphasize that this method is a fast and easy way to obtain model-free information about the structural properties of anisotropic scatterers, which we propose for example for the analysis of live data.

\section{Acknowledgements}

The SAXS experiments on the hematite spindles were performed on beamline ID02 at the European Synchrotron Radiation Facility (ESRF), Grenoble, France, and we are grateful to Sylvain Prévost for providing assistance. We thank the group of Magnetism and Magnetic Materials at the Universidad del País Vasco for preparing the magnetotactic bacteria and the Science and Technology Facilities Council (STFC) for access to the SAXS kit at the Materials Characterization Laboratory to perform the SAXS measurement.

\section{Funding information}

P. Bender acknowledges financial support from the National Research Fund of Luxembourg (CORE SANS4NCC grants), L. Marcano acknowledges the Basque Government for her fellowship (POS_2018_1_0070) and S. Disch acknowledges financial support from the German Research Foundation (DFG: DI 1788/2-1).

\section{References}

Adamo, M., Poulos, A. S., Lopez, C. G., Martel, A., Porcar, L. \& Cabral, J. T. (2018). Soft Matter, 14, 1759-1770.

Alves, C., Pedersen, J. S. \& Oliveira, C. L. P. (2017). J. Appl. Cryst. 50, 840-850.

Bender, P., Bogart, L. K., Posth, O., Szczerba, W., Rogers, S. E., Castro, A., Nilsson, L., Zeng, L. J., Sugunan, A., Sommertune, J., Fornara, A., González-Alonso, D., Barquín, L. F. \& Johansson, C. (2017). Sci. Rep. 7, 45990.

Bender, P., Günther, A., Honecker, D., Wiedenmann, A., Disch, S., Tschöpe, A., Michels, A. \& Birringer, R. (2015). Nanoscale, 7, 17122-17130.

Berkov, D. V., Görnert, P., Buske, N., Gansau, C., Mueller, J., Giersig, M., Neumann, W. \& Su, D. (2000). J. Phys. D Appl. Phys. 33, 331337.

Calabrese, M. A., Wagner, N. J. \& Rogers, S. A. (2016). Soft Matter, 12, 2301-2308.

Fritz-Popovski, G. (2013). J. Appl. Cryst. 46, 1447-1454.

Fritz-Popovski, G. (2015). J. Appl. Cryst. 48, 44-51.

Glatter, O. (1977). J. Appl. Cryst. 10, 415-421.

Glatter, O. (1979). J. Appl. Cryst. 12, 166-175.

Hansen, P. C. (1987). BIT, 27, 534-553.

Hansen, S. (2000). J. Appl. Cryst. 33, 1415-1421.

Heijden, P. C. van der, Rubatat, L. \& Diat, O. (2004). Macromolecules, 37, 5327-5336.

Hoffelner, D., Kundt, M., Schmidt, A. M., Kentzinger, E., Bender, P. \& Disch, S. (2015). Faraday Discuss. 181, 449-461.

Märkert, C., Fischer, B. \& Wagner, J. (2011). J. Appl. Cryst. 44, 441447.

Mettus, D. \& Michels, A. (2015). J. Appl. Cryst. 48, 1437-1450.

Michels, A. (2014). J. Phys. Condens. Matter, 26, 383201.

Michels, A., Viswanath, R. N., Barker, J. G., Birringer, R. \& Weissmüller, J. (2003). Phys. Rev. Lett. 91, 267204.

Nack, A., Seifert, J., Passow, C. \& Wagner, J. (2018). J. Appl. Cryst. 51, 87-96.

Narayanan, T., Sztucki, M., Van Vaerenbergh, P., Léonardon, J., Gorini, J., Claustre, L., Sever, F., Morse, J. \& Boesecke, P. (2018). J. Appl. Cryst. 51, 1511-1524.

Nielsen, S. S., Møller, M. \& Gillilan, R. E. (2012). J. Appl. Cryst. 45, 213-223. 
Orue, I., Marcano, L., Bender, P., García-Prieto, A., Valencia, S., Mawass, M., Gil-Cartón, D., Venero, D. A., Honecker, D., GarcíaArribas, A., Fernández Barquín, L., Muela, A. \& Fdez-Gubieda, M. (2018). Nanoscale, 10, 7407-7419.

Panine, P., Gradzielski, M. \& Narayanan, T. (2003). Rev. Sci. Instrum. 74, 2451-2455.

Pedersen, J. S. (1997). Adv. Colloid Interface Sci. 70, 171-210.

Poulos, A. S., Nania, M., Lapham, P., Miller, R. M., Smith, A. J., Tantawy, H., Caragay, J., Gummel, J., Ces, O., Robles, E. S. \& Cabral, J. T. (2016). Langmuir, 32, 5852-5861.

Qiu, X., Thompson, J. W. \& Billinge, S. J. L. (2004). J. Appl. Cryst. 37, 678.
Reufer, M., Dietsch, H., Gasser, U., Hirt, A., Menzel, A. \& Schurtenberger, P. (2010). J. Phys. Chem. B, 114, 4763-4769.

Roeder, L., Bender, P., Kundt, M., Tschöpe, A. \& Schmidt, A. M. (2015). Phys. Chem. Chem. Phys. 17, 1290-1298.

Stehle, R., Goerigk, G., Wallacher, D., Ballauff, M. \& Seiffert, S. (2013). Lab Chip, 13, 1529-1537.

Svergun, D. I. \& Koch, M. H. J. (2003). Rep. Prog. Phys. 66, 17351782.

Viereck, T., Draack, S., Schilling, M. \& Ludwig, F. (2019). J. Magn. Magn. Mater. 475, 647-651.

Zákutná, D., Falke, Y., Dresen, D., Prévost, S., Bender, P., Honecker, D. \& Disch, S. (2019). Nanoscale, 11, 7149-7156. 